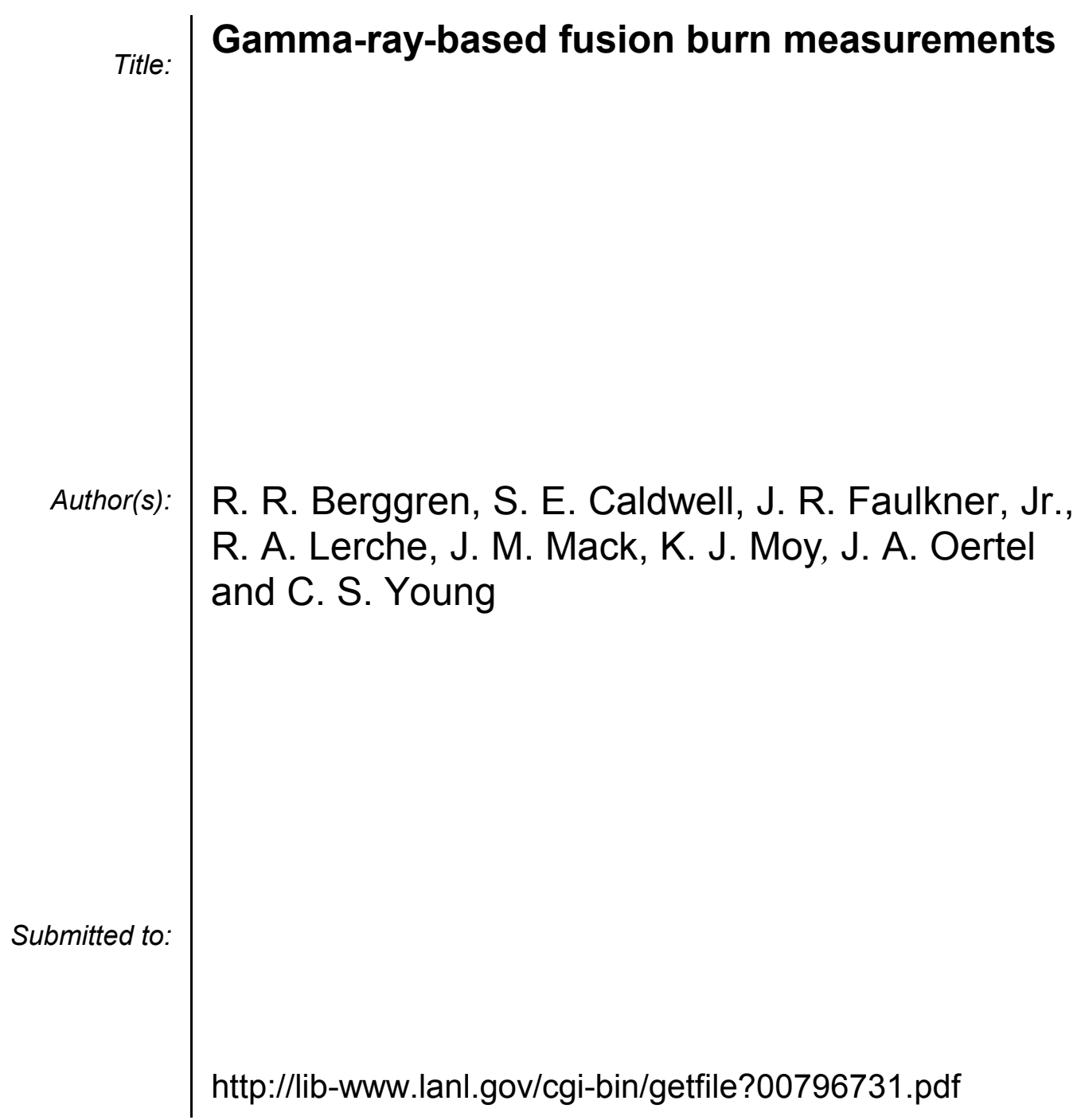

Los Alamos National Laboratory, an affirmative action/equal opportunity employer, is operated by the University of California for the U.S. Department of Energy under contract W-7405-ENG-36. By acceptance of this article, the publisher recognizes that the U.S. Government retains a nonexclusive, royaltyfree license to publish or reproduce the published form of this contribution, or to allow others to do so, for U.S. Government purposes. Los Alamos National Laboratory requests that the publisher identify this article as work performed under the auspices of the U.S. Department of Energy. Los Alamos National Laboratory strongly supports academic freedom and a researcher's right to publish; as an institution, however, the Laboratory does not endorse the viewpoint of a publication or guarantee its technical correctness. 


\title{
Gamma-ray-based fusion burn measurements
}

\author{
R. R. Berggren, S. E. Caldwell, and J. R. Faulkner, Jr. \\ Los Alamos National Laboratory, Los Alamos, New Mexico 87545 \\ R. A. Lerche \\ Lawrence Livermore National Laboratory \\ J. M. Mack \\ Los Alamos National Laboratory, Los Alamos, New Mexico 87545 \\ K. J. Moy \\ Bechtel Nevada \\ J. A. Oertel and C. S. Young ${ }^{\text {a) }}$ \\ Los Alamos National Laboratory, Los Alamos, New Mexico 87545
}

(Presented on 20 June 2000)

A gas Cerenkov detector with a $12-\mathrm{MeV}$ threshold for gamma-ray detection has been built for use on the OMEGA laser system to record high-energy gamma rays emitted during DT gas burn. Recording the $16.7-\mathrm{MeV}$ gamma ray while discriminating against the lower energy 14-MeV neutron-induced gammas is an important objective using this detector system. Detector design, sensitivity, and background studies were possible using the Integrated Tiger Series Monte Carlo code modified to include Cerenkov production and full time-history of all particles. The results of this code were iterated with the ASAP optics code to optimize the light collection system, while providing the radiation shielding and stray light baffles to minimize backgrounds. As an initial test of the instrument, $8-20 \mathrm{MeV}$ electrons from the Idaho State University linear accelerator were used in lieu of gamma rays. The primary results of these tests are that electron-produced Cerenkov has been observed and the Cerenkov threshold curve established for this instrument. () 2001 American Institute of Physics. [DOI: 10.1063/1.1321003]

\section{INTRODUCTION}

DT fusion gamma rays were observed using high-yield NOVA capsules. ${ }^{1}$ An important conclusion was that a gas Cerenkov detector (GCD) system could provide better time resolution of the 16.7-MeV gamma ray. Energy thresholding would substantially eliminate possible distortion of the burn signal by time-of-flight effects of concurrent $14-\mathrm{MeV}$ neutron-induced gamma rays. Most of the potentiallyinterfering 14-MeV neutron-induced signal comes from relatively low-energy neutron-inelastic gammas $(<10 \mathrm{MeV})$ in the laser target materials; it is greatly reduced by choosing a threshold at $12 \mathrm{MeV}$. A small residual interference is due to the relatively improbable neutron-capture gamma rays that extend as high as $25 \mathrm{MeV}$ in energy.

Cerenkov light ${ }^{2}$ is produced by particles traveling faster than $c / n$ (the speed of light divided by the index of refraction of the material through which the particle is traveling). $\varepsilon$, which is $n-1$, is proportional to the density and hence the pressure of the gas. Adjustment of gas pressure is used to discriminate against lower energy particles. Figure 1 shows how the detection threshold varies with gas pressure for $\mathrm{CO}_{2}$. A pressure of 27.3 psia $\left(\right.$ at $0{ }^{\circ} \mathrm{C}$ ) yields an electron threshold energy of $12 \mathrm{MeV}$; the corresponding value for $n$ is 1.000 83. The Cerenkov spectrum is proportional to $1 / \lambda^{2}$

${ }^{\text {a)} E l e c t r o n i c ~ m a i l: ~ c s y o u n g ~ @ l a n l . g o v ~}$ (where $\lambda$ is the wavelength) and hence transmission and detector sensitivity in the near ultraviolet are important.

$\mathrm{A} \mathrm{CO}_{2}$ Cerenkov detector (Fig. 2) has been built which can be inserted into a ten-inch manipulator (TIM) on the OMEGA laser system to record high-energy gamma rays. The detector first converts gamma rays to electrons through Compton conversion and pair production; then electrons above the $\mathrm{CO}_{2}$ gas threshold emit Cerenkov photons. A light collection system (including the gamma and electron path-

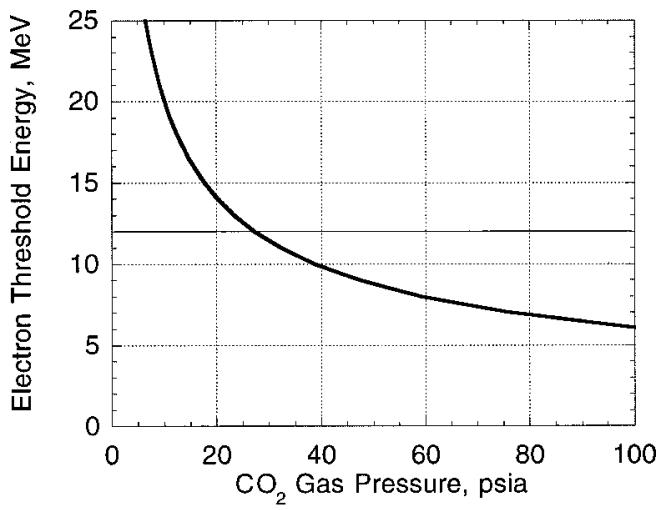

FIG. 1. Cerenkov production threshold for $\mathrm{CO}_{2}$. The electron energy required to produce Cerenkov light in $\mathrm{CO}_{2}$ is determined by the index of refraction which is a function of the gas pressure. 


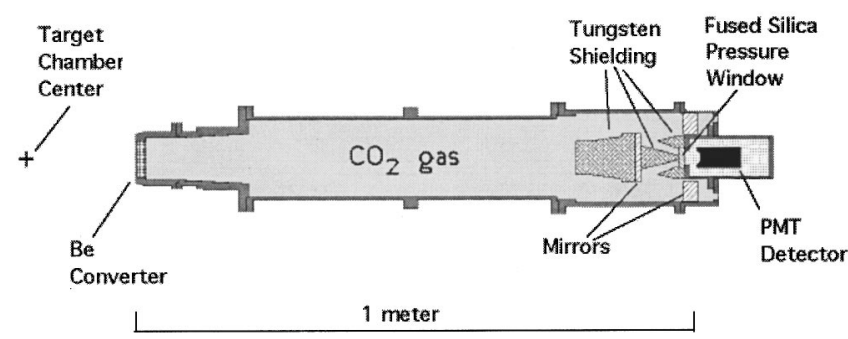

FIG. 2. Omega detector schematic. At Omega, gammas will originate at the target on the left. Compton conversion and pair production take place within the $\mathrm{Be}$ converter and to some extent within the $\mathrm{CO}_{2}$ gas. Cerenkov light is produced within the gas by electrons with energies above the $12 \mathrm{MeV}$ threshold. Cerenkov light is collected by the two-mirror telescope and directed to the PMT. For electron tests at ISU the Be converter was removed and electrons penetrated the remaining $1 \mathrm{~mm} \mathrm{Al}$ from the left.

length but neglecting the photomultiplier) has been designed such that the theoretical time resolution is $<10 \mathrm{ps}$.

Observation of the $16.7-\mathrm{MeV}$ gamma $^{3}$ is the primary objective during this phase of detector development. The initial time response goal is 200-300 ps, set by the bandwidth of the microchannel plate (MCP) photomultiplier detectors (PMT). This bandwidth is sufficient to differentiate fusion gamma rays from laser and most neutron-induced radiation which will be temporally separated from the burn signal by more than 300 ps. The next phase of detector development will focus on high bandwidth recording of the Cerenkov signal by replacing the photomultiplier with a streak camera.

Simulation of such systems involves the production and transport of neutrons, gammas, electrons, and Cerenkov photons. New code development was directed toward the inclusion of time-dependent Cerenkov photon production, as coupled to the photon/electron cascade, and the subsequent optical ray-trace of Cerenkov photons through the threedimensional Monte Carlo (MC) geometries. The foundation used is that of the ACCEPT code of the Integrated Tiger Series, Version 3.0 (ITS), ${ }^{4}$ and the details of the Monte Carlo model development are presented elsewhere. ${ }^{5}$

This article describes the design of the detector and the initial test of the instrument with electrons. More realistic tests of the GCD will be carried out at the University of Rochester's OMEGA laser facility.

\section{OPTICAL DESIGN}

Several spherical and elliptical gas cell designs for OMEGA have been studied to maximize fusion gamma sensitivity, while minimizing the inelastic gamma sensitivity. An elliptical design was originally pursued, ${ }^{5}$ but a spherical system proved to be the design of choice. An optomechanical design effort was undertaken to (1) produce a design for a system that could be easily installed in the OMEGA laser; (2) optimize the collection efficiency; and (3) minimize sources of light that might interfere with the measurement.

Ease of installation and operation is provided if the instrument is designed for use in one of the OMEGA TIMs. They facilitate insertion and removal of the device without disturbing the chamber vacuum and they also allow the in- strument placement close to the target chamber center (TCC). The converter was then assumed to be as large and as close to the TCC as possible, limited by the need to stay within an f/7 cone to prevent interference with the laser beams. The need to time separate gammas from 14-MeV neutrons dictated limiting the target-converter distance to about $20 \mathrm{~cm}$.

The Monte Carlo results give the phase-space distributions of Cerenkov photons in the gas for a given pencil beam (or other source configuration) of fusion gamma rays incident on the converter. These Cerenkov photon data were then imported into the ASAP optical analysis code ${ }^{6}$ and ray traced through the instrument geometry. Iterations proceeded resulting in an optimized light collection design.

Various optical systems were tried in order to maximize collection efficiency. A class of nonimaging systems can sometimes give improved collection, particularly for uniform sources of limited angular extent. In our case the Monte Carlo code showed that ray generation falls off with angle so that a conventional imaging system was equally effective. For an imaging system, the image and the object planes are reciprocal. Thus, even though the Cerenkov source is a volume source, the PMT is at the image of a fixed plane. Backtracking the Cerenkov photon trajectories showed that the pattern was most dense at a plane near the converter. Therefore, maximum collection efficiency could be obtained by imaging the converter onto the PMT photocathode.

A conventional two-mirror system, similar to a Cassegrain telescope but with a flat secondary, gave the best results. By placing a thick tungsten block behind the secondary mirror, gamma rays could be prevented from directly reaching the PMT. Furthermore, the Cassegrain configuration means that light rays have a significant extra transit time as they are reflected from the primary and back to the secondary before reaching the PMT. Thus the Cerenkov signal would be separated advantageously from light produced by other gammas or electrons directly reaching the PMT.

\section{SIGNAL SIMULATION}

With the anticipated optical design, the Monte Carlo simulations proceeded tracking the time/position histories of all particles. The sensitivity of the detector to both gammas and electrons incident on the front of the detector was studied.

These simulations predict a signal at approximately 500 ps before the arrival of the Cerenkov photons from the gas. The early signal is due to Cerenkov photons from the fused silica pressure window and the detector's fused silica vacuum window. The windows (index of refraction of $\sim 1.5$ ) have a Cerenkov production threshold of $\sim 180 \mathrm{keV}$. Highenergy electrons or gammas scattering from the lateral walls of the $\mathrm{CO}_{2}$ pressure chamber have a relatively direct path to the detector region. Subsequent implementations of the GCD will avoid this problem by placing the offending elements in an easily shielded location, but for this TIM application, careful shielding of the exposed windows (see Fig. 2) was necessary. Iteration between the Monte Carlo and optical codes was used to determine the placement of tungsten 


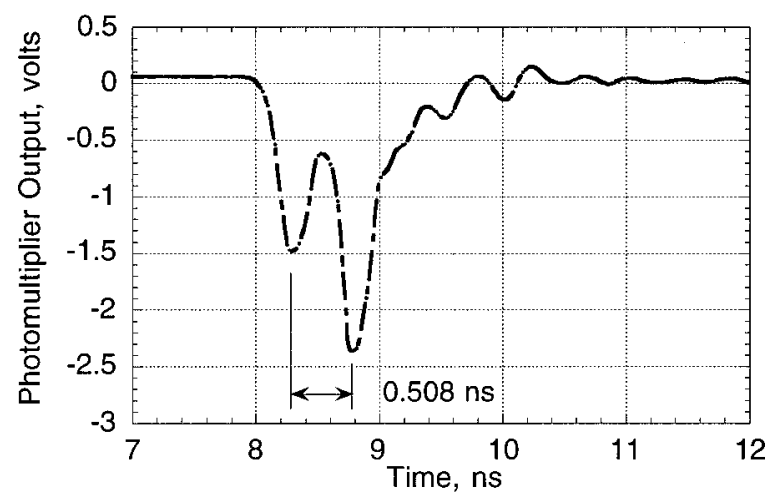

FIG. 3. The PMT signal at $13.5 \mathrm{MeV}$ from the Tektronix SCD-5000 recorder. The first pulse is due to scattered radiation reaching the PMT region. The second pulse is the Cerenkov light from the gas which has a longer pathlength within the gas cell because of the dual mirror telescope. At this energy, the Cerenkov light has been attenuated by about $60 \times$ with ND filters.

shielding necessary to minimize these background signals with minimal decrease in the optical signal.

\section{INITIAL TESTS}

Initial tests were run using electrons from the Idaho Accelerator Center (Pocatello, ID), where the Idaho State University maintains an electron linear accelerator (LINAC) capable of producing mono-energetic, 50-ps wide electron pulses at rep rates of up to $120 \mathrm{~Hz}$. With the Be converter removed from the GCD the 6-mm diameter electron beam could penetrate the remaining $1-\mathrm{mm} \mathrm{Al}$ window and enter the gas cell with little energy degradation $(\sim 0.5 \mathrm{MeV})$. Data at electron energies of 8-20 MeV were acquired with a Photek PMT113 detector operating at $-3 \mathrm{kV} \quad(\mathrm{QE}=260$ electrons/photon at $400 \mathrm{~nm}$ ). The beam energy spread is not currently well known but is estimated to be about 3\%. Typical values for the $\mathrm{CO}_{2}$ gas pressure and temperature were 30 psia and $29^{\circ} \mathrm{C}$, respectively. A $15-\mathrm{cm}$ lead wall was placed between the LINAC and the Cerenkov detector to provide shielding of the detector and also collimate the electron beam to about $6 \mathrm{~mm}$. At each energy, the detector and LINAC beam monitor were recorded simultaneously for each of three or more LINAC pulses. Subsequent runs with a Faraday cup calibrated the beam monitor at each energy. Typical data for electrons of $13.5 \mathrm{MeV}$ appear in Fig. 3 and the energy scan from 8 to $20 \mathrm{MeV}$ appears in Fig. 4.

At electron energies well above threshold, neutral density (ND) filters were inserted between the pressure window and the detector to reduce the intensity of gas Cerenkov light. For the data of Fig. 3 at $13.5 \mathrm{MeV}$ the ND attenuation was about 60 . The apparent time separation of $\sim 500 \mathrm{ps}$ is in accord with the calculated precursor time. At $16 \mathrm{MeV}$, an opaque foil was inserted between the pressure window and the PMT with the result that the second signal went away but the first did not change measurably. This indicates the dominant source of the precursor is the photomultiplier window due to either Cerenkov in the fused silica or possibly electrons at the input to the MCP of the PMT and not the pres-

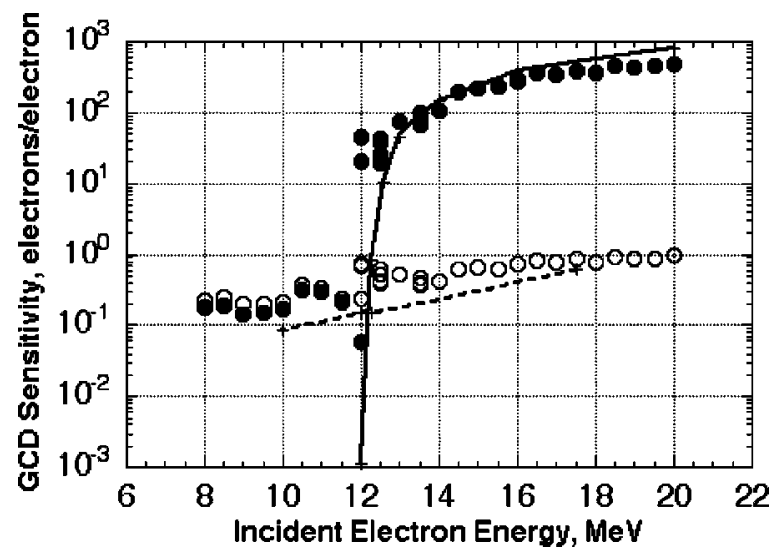

FIG. 4. Comparison of preliminary LINAC data and simulation for electrons from the PMT detector per incident electron. The plotted lines are from the Monte Carlo simulations. The solid line is the calculated gas Cerenkov contribution and the dashed line is the calculated precursor due to Cerenkov in the fused silica windows. The plotted points are LINAC data: the solid circles are the delayed "gas"' signal (second pulse in Fig. 3) and the open circles are the earlier occurring signal from the PMT window (first signal in Fig. 3).

sure window. When the $\mathrm{CO}_{2}$ gas was removed from the chamber the second signal disappeared, indicating the second signal comes from the gas.

The energy dependence of the signal from the $\mathrm{CO}_{2}$ gas and the precursor are shown in Fig. 4 in terms of the PMT detector charge per unit LINAC beam charge. Observed values near the threshold energy of $12 \mathrm{MeV}$ are uncertain presumably due to the poor energy definition and/or repeatability of the LINAC beam for these initial measurements. Nevertheless, the threshold is apparent; the difference in sensitivity between 16.75 - and $11.5-\mathrm{MeV}$ electrons is about three orders of magnitude. Away from the threshold the apparent scatter is probably indicative of the experimental error. For comparison, Fig. 4 also shows the calculated gas Cerenkov signal and calculated precursor due to the fused silica windows. The observed gas signal below $12 \mathrm{MeV}$ is partially transition radiation, which is not part of the current calculation. For the absolute comparison of Fig. 4 the number of calculated photons was multiplied by the measured PMT quantum efficiency (electrons per photon) weighted by the assumed $1 / \lambda_{2}$ wavelength dependence of the Cerenkov light. Aside from the threshold region and some difference in slope above threshold, agreement between calculation and theory for the gas Cerenkov is quite good.

The difference between the magnitudes of calculated and observed precursors indicates that there are sources of precursor other than those in the calculation. One source not included in the calculation is the effect of scattered electrons or gamma-rays impinging on the micro-channel plate.

\section{CONCLUSIONS}

Preliminary tests show that the GCD responds to electrons as expected. The Monte Carlo simulations have been partially validated for this instrument by predicting the threshold capability of the detector threshold and the timing 
of the background. Further work is required for an absolute comparison between observed and computed detector signals.

The NIF requirements for high bandwidth $(>20 \mathrm{Ghz})$ burn-history diagnostics and greater detector setback $(\geqslant 50$ $\mathrm{cm}$ ) can be met using gamma-ray-based measurements. Use of an electron LINAC beam allowed an operational assessment of this gas Cerenkov detector design and provided an excellent model verification/validation tool.

\section{ACKNOWLEDGMENTS}

The authors would like to thank the staff and management of the Idaho Accelerator Center for providing the test facilities for this project. In particular, Ron Sturges (Bechtel Nevada), Mel Williams (IAC), Joe Garcia (LANL) and Dave
Schwellenbach (ISU) provided the support needed to carry out these tests. This work is funded by the U.S. Department of Energy.

${ }^{1}$ S. E. Caldwell, S. S. Han, J. R. Joseph, T. L. Petersen, and C. S. Young, Rev. Sci. Instrum. 68, (1997).

${ }^{2}$ J. V. Jelley, Cerenkov Radiation (Pergamon, New York, 1958).

${ }^{3}$ S. S. Medley, F. E. Cecil, D. Cole, M. A. Conway, and F. J. Wilkinson III, Rev. Sci. Instrum. 56, 975 (1985).

${ }^{4}$ J. A. Halbleib and T. A. Mehlhorn, ITS: The Integrated TIGER Series of Coupled Electron/Photon Monte Carlo Transport Codes, Sandia National Laboratories, Albuquerque, NM, Sandia Report: SAND84-0573, 1984.

${ }^{5}$ J. M. Mack and C. S. Young, "Monte Carlo Simulation of Highbandwidth Cerenkov Detectors for NIF Fusion Burn Measurements," First International Conference On Inertial Fusion Sciences and Applications, ENSCPB, University Bordeaux 1, France, 12-17 September 1999.

${ }^{6} \mathrm{ASAP}$ is a product of Breault Research Organization, Tucson, AZ. 